\title{
Irregularity strength of regular graphs
}

\author{
Jakub Przybyło \\ AGH University of Science and Technology \\ Al. Mickiewicza 30, 30-059 Kraków, Poland \\ przybylo@wms.mat.agh.edu.pl \\ Submitted: Nov 12, 2007; Accepted: Jun 9, 2008; Published: Jun 13, 2008 \\ Mathematics Subject Classifications: 05C78
}

\begin{abstract}
Let $G$ be a simple graph with no isolated edges and at most one isolated vertex. For a positive integer $w$, a $w$-weighting of $G$ is a map $f: E(G) \rightarrow\{1,2, \ldots, w\}$. An irregularity strength of $G, s(G)$, is the smallest $w$ such that there is a $w$-weighting of $G$ for which $\sum_{e: u \in e} f(e) \neq \sum_{e: v \in e} f(e)$ for all pairs of different vertices $u, v \in$ $V(G)$. A conjecture by Faudree and Lehel says that there is a constant $c$ such that $s(G) \leq \frac{n}{d}+c$ for each $d$-regular graph $G, d \geq 2$. We show that $s(G)<16 \frac{n}{d}+6$. Consequently, we improve the results by Frieze, Gould, Karoński and Pfender (in some cases by a $\log n$ factor) in this area, as well as the recent result by Cuckler and Lazebnik.
\end{abstract}

Keywords: irregularity strength, graph weighting, regular graph

\section{Introduction}

All graphs we consider are simple and finite. An edge $\{u, v\}$ will be denoted by $u v$ or $v u$ for short at times. For a given graph $G$ and its vertex $v, N_{G}(v) d_{G}(v), V(G), E(G)$ and $\delta(G)$ (or simply $N(v), d(v), V, E$ and $\delta$ ) denote the set of neighbours and the degree of $v$ in $G$, the set of vertices, the set of edges and the minimum degree of $G$, respectively. By $G[D]$ we mean an induced subgraph of $G$ with the vertex set $D \subseteq V(G)$. A set $\mathcal{V}=\left\{V_{1}, V_{2}, \ldots, V_{k}\right\}$ of disjoint subsets of a set $V$ is called a partition of $V$ if the union of all elements of $\mathcal{V}$ is $V$ and $V_{i} \neq \emptyset$ for every $i$. We shall denote as $P_{k}$ a path of length $k-1$ and write $P_{k}=v_{1} v_{2} \ldots v_{k}$ for short if $v_{i} v_{i+1}$ are its consecutive edges, $i=1,2, \ldots, k-1$.

For a graph $G$ and a finite set $S$ of integers, an $S$-weighting of $G$ is an assignment $f: E(G) \rightarrow S$. If $S=\{1,2, \ldots, w\}$, then we call $f$ a $w$-weighting of $G$. Moreover, $f(e)$ is called the weight of an edge $e \in E(G)$, while the weight of $v \in V(G)$ is defined as $f(v)=\sum_{u \in N(v)} f(v u)$. A weighting $f$ is irregular if the obtained weights of all vertices are different. The smallest positive integer $w$ for which there exists an irregular $w$-weighting 
of $G$ is called the irregularity strength of $G$ and is denoted by $s(G)$. If it does not exist, we write $s(G)=\infty$. It is easy to see that $s(G)<\infty$ iff $G$ contains no isolated edges and at most one isolated vertex.

The notion of the irregularity strength was introduced by Chartrand at al. [3]. It was motivated by the well known fact that a simple graph of order at least 2 must contain a pair of vertices with the same degree. On the other hand, a multigraph can be irregular, i.e. the degrees of its vertices can all be distinct. Now suppose we want to multiply the edges of a graph $G$ in order to create an irregular multigraph of it. Then $s(G)$ is equal to the smallest maximum multiplicity of an edge in such a multigraph, see [7] for a survey by Lehel on this parameter. We will focus our attention on the regular graphs, which (not only by the name) seem to be the most difficult to be "made irregular". A simple counting argument, see e.g. [3], shows that $s(G) \geq\left\lceil\frac{n+d-1}{d}\right\rceil$ for all $d$-regular graphs, $d \geq 2$, of order $n$. A question whether maybe just "a few" more weights than this lower bound would always suffice was posed by Jacobson (see [7]) after obtaining a number of supporting arguments. This was formulated as a conjecture by Faudree and Lehel.

Conjecture 1 ([5]) There exists an absolute constant $c$ such that

$$
s(G) \leq \frac{n}{d}+c
$$

for each d-regular graph $G, d \geq 2$, of order $n$.

They also showed the following.

Theorem 2 ([5]) Let $G$ be a d-regular graph, $d \geq 2$, of order $n$. Then

$$
s(G) \leq\left\lceil\frac{n}{2}\right\rceil+9 .
$$

About 15 years later a sizeable step forward in the survey on this problem was made by Frieze, Gould, Karoński and Pfender.

Theorem 3 ([6]) Let $G$ be a d-regular graph of order $n$ with no isolated vertices or edges.

(a) If $d \leq\left\lfloor(n / \ln n)^{1 / 4}\right\rfloor$, then $s(G) \leq 10 n / d+1$,

(b) If $\left\lfloor(n / \ln n)^{1 / 4}\right\rfloor+1 \leq d \leq\left\lfloor n^{1 / 2}\right\rfloor$, then $s(G) \leq 48 n / d+1$,

(c) If $d \geq\left\lfloor n^{1 / 2}\right\rfloor+1$, then $s(G) \leq 240(\log n) n / d+1$.

Their result was recently supplemented (and improved in some cases) by Cuckler and Lazebnik.

Theorem 4 ([4]) Let $G$ be a d-regular graph of order $n$ with no isolated vertices or edges. If $d \geq 10^{4 / 3} n^{2 / 3} \log ^{1 / 3} n$, then $s(G) \leq 48 n / d+6$.

Unfortunately, these results do not confirm even a weaker form of Conjecture 1, namely that

$$
s(G) \leq c_{1} \frac{n}{d}+c_{2}
$$


holds for all $d$-regular graphs of order $n$, with $c_{1}$ and $c_{2}$ being absolute positive constants. In other words, we do not even know if $s(G)$ is of order $\frac{n}{d}$ suggested in this conjecture (see Theorem $3(\mathrm{c})$ ). We will show it quite briefly in the next section, see Corollary 10. Then we will improve the obtained constants $c_{1}, c_{2}$ by a careful construction and prove the following main result of the paper in the last section.

Theorem 5 Let $G$ be a d-regular graph of order $n$ with no isolated vertices or edges. Then

$$
s(G)<16 \frac{n}{d}+6 .
$$

\section{The right order of $s(G)$}

Let $g$ be a $w$-weighting of a graph $G$ and let us define

$$
m_{g}=\max _{X \subseteq V(G)}\{|X|: g(u)=g(v) \text { for all } u, v \in X\} .
$$

The main idea of the proof of Theorem 3 relied on two steps. First the authors found a $w$-weighting $g$ with small $m_{g}$ and small $w$, e.g. $w=2$, using probabilistic tools. Then they modified $g$ to an irregular assignment by means of the following deterministic lemma.

Lemma 6 ([6]) Let $G$ be a d-regular graph without isolated vertices or isolated edges, and let $g$ be a w-weighting of $G$. Then, there exists an irregular $\left((3 w-1) m_{g}+1\right)$-weighting of $G$.

Our approach, which will be explained in details later, is in a way similar. An equivalent of the first step described will be Corollary 11, which we prove at the beginning of the third section. It will be responsible for grouping the set of vertices into fairly small subsets of elements with the same weight. Our main tool will be the following theorem by Addario-Berry, Dalal and Reed.

Theorem 7 ([1]) Given a graph $G$ and for all $v \in V(G)$, integers $a_{v}^{-}, a_{v}^{+}$such that $a_{v}^{-} \leq\left\lfloor\frac{d(v)}{2}\right\rfloor \leq a_{v}^{+}<d(v)$, and

$$
a_{v}^{+} \leq \min \left(\frac{d(v)+a_{v}^{-}}{2}+1,2 a_{v}^{-}+3\right)
$$

there exists a spanning subgraph $H$ of $G$ such that $d_{H}(v) \in\left\{a_{v}^{-}, a_{v}^{-}+1, a_{v}^{+}, a_{v}^{+}+1\right\}$ for all $v \in V(G)$.

Corollary 8 Let $d>0$ be an integer. There exists a set $S^{-}$of $\left\lceil\frac{d}{4}\right\rceil$ consecutive integers such that given any d-regular graph $G$ and numbers $a_{v}^{-} \in S^{-}, a_{v}^{+}:=a_{v}^{-}+\left\lceil\frac{d}{4}\right\rceil+1$ for each $v \in V(G)$, there exists a spanning subgraph $H$ of $G$ such that $d_{H}(v) \in\left\{a_{v}^{-}, a_{v}^{-}+\right.$ $\left.1, a_{v}^{+}, a_{v}^{+}+1\right\}$ for all $v \in V(G)$. 
Proof. The theorem is obvious for $d \leq 3$, so let $d \geq 4$. Assume first that $d$ is not divisible by 4 and take $S^{-}:=\left\{\left\lceil\frac{d}{4}\right\rceil-2, \ldots, 2\left\lceil\frac{d}{4}\right\rceil-3\right\}$. Clearly $\left|S^{-}\right|=\left\lceil\frac{d}{4}\right\rceil$ and for $a_{v}^{-} \in S^{-}, a_{v}^{+}:=a_{v}^{-}+\left\lceil\frac{d}{4}\right\rceil+1$, we have $a_{v}^{-} \leq 2\left\lceil\frac{d}{4}\right\rceil-3 \leq\left\lfloor\frac{d}{2}\right\rfloor,\left\lfloor\frac{d}{2}\right\rfloor \leq 2\left\lceil\frac{d}{4}\right\rceil-1 \leq a_{v}^{+}$and $a_{v}^{+} \leq 3\left\lceil\frac{d}{4}\right\rceil-2<d$, hence, by Theorem 7 , it is enough to prove (4) for all $v \in V(G)$. Note then that $a_{v}^{+}=a_{v}^{-}+\left\lceil\frac{d}{4}\right\rceil+1 \leq a_{v}^{-}+a_{v}^{-}+3$ and $a_{v}^{+}=\frac{a_{v}^{-}}{2}+\frac{a_{v}^{-}}{2}+\left\lceil\frac{d}{4}\right\rceil+1 \leq$ $\frac{a_{v}^{-}}{2}+\left\lceil\frac{d}{4}\right\rceil-\frac{3}{2}+\left\lceil\frac{d}{4}\right\rceil+1 \leq \frac{a_{v}^{-}}{2}+\frac{d}{2}+1$, thus (4) holds.

Analogously, if $d$ is divisible by 4 , we can take $S^{-}:=\left\{\frac{d}{4}, \ldots, \frac{d}{2}-1\right\}$.

Let $G=(V, E)$ be a graph and let $A, B$ be two nonempty, nonintersecting subsets of $V$. For a given weighting $f$ of edges of $G$, let $d_{f}(A, B):=\min \{|f(v)-f(w)|: v \in A, w \in B\}$ denote the distance between $A$ and $B$ with respect to $f$. Moreover, let $d_{f}(A):=0$ if $f$ is constant on $A$ or $d_{f}(A):=\min \{|f(v)-f(w)|: v, w \in A, f(v) \neq f(w)\}$ otherwise.

Corollary 9 For each d-regular graph $G$ and a partition $\left\{A_{1}, \ldots, A_{\left\lceil\frac{d}{8}\right]}\right\}$ of its vertices, there exists a 2-weighting $f$ of $G$ such that $d_{f}\left(A_{i}, A_{j}\right) \geq 1$ for $i \neq j$.

Proof. Let $G=(V, E)$ be a $d$-regular graph with $d>0$ and let $\left\{A_{1}, \ldots, A_{\left\lceil\frac{d}{8}\right\rceil}\right\}$ be any partition of $V$. Let $S^{-}=\left\{s_{1}, \ldots, s_{\left\lceil\frac{d}{4}\right\rceil}\right\}$ be an appropriate set from Corollary 8, where $s_{1}, \ldots, s_{\left\lceil\frac{d}{4}\right\rceil}$ are $\left\lceil\frac{d}{4}\right\rceil$ consecutive integers. Let $a_{v}^{-}:=s_{2 i-1}$ for each $v \in A_{i}, i=1, \ldots,\left\lceil\frac{d}{8}\right\rceil$ (hence $s_{1} \leq a_{v}^{-} \leq s_{\left\lceil\frac{d}{4}\right\rceil}$ for all $v \in V$ ). By Corollary 8, there exists a spanning subgraph $H$ of $G$ such that $d_{H}(v) \in\left\{s_{2 i-1}, s_{2 i-1}+1, s_{2 i-1}+\left\lceil\frac{d}{4}\right\rceil+1, s_{2 i-1}+\left\lceil\frac{d}{4}\right\rceil+2\right\}=: S_{i}$ for every $v \in A_{i}, i=1 \ldots,\left\lceil\frac{d}{8}\right\rceil$. Note that since $s_{\left\lceil\frac{d}{4}\right\rceil}+1<s_{1}+\left\lceil\frac{d}{4}\right\rceil+1$, then $S_{i} \cap S_{j}=\emptyset$ for $i \neq j$. Therefore, if we set $f(e)=2$ for all the edges of the subgraph $H$ and $f(e)=1$ for all the other edges of $G$, then $|f(v)-f(w)| \geq 1$ whenever $v \in A_{i}, w \in A_{j}$ and $i \neq j$ (because $G$ is a regular graph).

An almost immediate consequence of the above corollary is the following one, which confirms that (3) holds.

Corollary 10 Let $G$ be a d-regular graph of order $n$ with no isolated vertices or edges. Then

$$
s(G)<40 \frac{n}{d}+11 .
$$

Proof. Take any partition $\left\{A_{1}, \ldots, A_{\left\lceil\frac{d}{8}\right\rceil}\right\}$ of $V(G)$ such that $\left|A_{i}\right| \leq 2\left\lceil\frac{4 n}{d}\right\rceil$ for all $i$ (it exists since $\left.\left\lceil\frac{d}{8}\right\rceil\left(2\left\lceil\frac{4 n}{d}\right\rceil\right) \geq n\right)$. Then, by Corollary 9 , there is a 2-weighting $f$ of $G$ such that

$$
m_{f} \leq \max _{1 \leq i \leq\left\lceil\frac{d}{8}\right\rceil}\left|A_{i}\right| \leq 2\left\lceil\frac{4 n}{d}\right\rceil,
$$

hence, by Lemma 6, we have

$$
s(G) \leq 5\left(2\left\lceil\frac{4 n}{d}\right\rceil\right)+1<40 \frac{n}{d}+11 .
$$

This corollary already improves in many cases the results by Frieze at al., as well as the one by Cuckler and Lazebnik, see Theorems 3 and 4. 


\section{$3 \quad$ Improving the upper bound in (5)}

The rest of the paper is devoted to strengthening the inequality (5) above, i.e. replacing constants 40 and 11 by 16 and 6 . Our approach consists also of two steps, which very roughly look as follows. First we construct a weighting $f$ of a given graph $G$ that partition the vertex set into "small" subsets of vertices with the same weights, but in such a way that there is quite a big difference between the weights of vertices from distinct subsets. This will be provided by Corollary 11 below, which is an immediate consequence of Corollary 9 . Then we construct a weighting $g$, which is responsible for "scattering the weights" of the vertices from the subsequent subsets "not too far" from their initial weights, but in such a way that as a result they all have distinct weights. This is done in Lemma 15. The sum of this two weightings will be the desired one.

Corollary 11 For each d-regular graph $G$ and a partition $\left\{A_{1}, \ldots, A_{\left\lceil\frac{d}{8}\right\rceil}\right\}$ of its vertices, there exists a weighting $f: E(G) \rightarrow\left\{\left\lceil\frac{4 n}{d}\right\rceil+1,3\left\lceil\frac{4 n}{d}\right\rceil+2\right\}$ such that $d_{f}\left(A_{i}, A_{j}\right) \geq 2\left\lceil\frac{4 n}{d}\right\rceil+1$ for $i \neq j$ and $d_{f}\left(A_{i}\right)=0$ or $d_{f}\left(A_{i}\right) \geq 2\left\lceil\frac{4 n}{d}\right\rceil+1$ for all $i$.

Proof. Let $G=(V, E)$ be a $d$-regular graph with $d>0$ and let $\left\{A_{1}, \ldots, A_{\left\lceil\frac{d}{8}\right\rceil}\right\}$ be a partition of $V$. By Corollary 9, there is a 2-weighting $h$ of $G$ such that $d_{h}\left(A_{i}, A_{j}\right) \geq 1$ for $i \neq j$. Then it is enough to set $f(e)=\left\lceil\frac{4 n}{d}\right\rceil+1$ if $h(e)=1$ and $f(e)=3\left\lceil\frac{4 n}{d}\right\rceil+2$ if $h(e)=2$. Note that $\left(3\left\lceil\frac{4 n}{d}\right\rceil+2\right)-\left(\left\lceil\frac{4 n}{d}\right\rceil+1\right)=2\left\lceil\frac{4 n}{d}\right\rceil+1$. Therefore $|f(u)-f(v)|=0$ or $|f(u)-f(v)| \geq 2\left\lceil\frac{4 n}{d}\right\rceil+1$ for $u, v \in V$, since $G$ is a regular graph. Consequently, $d_{f}\left(A_{i}\right)=0$ or $d_{f}\left(A_{i}\right) \geq 2\left\lceil\frac{4 n}{d}\right\rceil+1$ for each $i$ by the definition of $d_{f}\left(A_{i}\right)$, and $d_{f}\left(A_{i}, A_{j}\right) \geq 2\left\lceil\frac{4 n}{d}\right\rceil+1$ for $i \neq j$ by Corollary 9 .

Let $P_{3}^{o}=v_{1} v_{2}^{o} v_{3}$ denote a path $P_{3}=v_{1} v_{2} v_{3}$ after removing a middle vertex $v_{2}$ from it, but without removing any edge. In other words, if $P_{3}=(V, E)$ is regarded as a graph $\left(V=\left\{v_{1}, v_{2}, v_{3}\right\}, E=\left\{v_{1} v_{2}, v_{2} v_{3}\right\}\right)$, then $P_{3}^{o}$ is an ordered pair $\left(V \backslash\left\{v_{2}\right\}, E\right)$. We shall call $P_{3}^{o}$ an open path of length 2 and $v_{2}^{o}$ will be referred to as an open vertex in $P_{3}^{o}$. The other vertices of $P_{3}^{o}$, as well as all the vertices of simple paths, e.g. $P_{2}, P_{3}$, will be called closed. We shall also abuse a little bit the established notation and call $P_{3}^{o}$ a graph (or a subgraph). Now, a $\left\{P_{2}, P_{3}, P_{3}^{o}\right\}$-factor of a graph $G$ is a collection of vertex (and edge) disjoint subgraphs of $G$ which are either paths of lengths 1 or 2, or open paths of length 2 (we call them the components of the factor), and that together span $G$. (If two graphs share only one vertex which is open in one or both of them, they are vertex disjoint.) Span here means that each vertex of $V(G)$ is a closed vertex of exactly one component of this factor. In this sense, e.g. each star (except $K_{1}$ ) has a $\left\{P_{2}, P_{3}, P_{3}^{o}\right\}$-factor.

Let $F$ be a forest. Denote by $c_{F}$ the number of components of $F$, by $L(F)$ the set of leaves of $F$ and let $R(F)=V(F) \backslash L(F)$.

In order to construct the weighting $g$ mentioned at the beginning of this section (and described in Lemma 15) we shall need a $\left\{P_{2}, P_{3}, P_{3}^{o}\right\}$-factor of a given graph $G$ consisting of not too many $P_{3}$ 's and sufficiently many $P_{3}^{o}$ 's, see Lemma 14 . To obtain it, we first prove the existence of a spanning forest $F$ of $G$ with "relatively small" value of $|R(F)|$, 
see Lemma 13. For this aim we shall use the domination number of $G, \gamma(G)$, which is the size of the smallest dominating set of $G$, i.e. the subset, say $D$, of $V(G)$ such that each vertex in $V(G) \backslash D$ has a neighbour in $D$. The following probabilistic result can be found in Alon and Spencer [2].

Theorem 12 ([2]) Let $G$ be a graph of order $n$ and with $\delta(G) \geq 2$. Then

$$
\gamma(G) \leq \frac{n(1+\ln (\delta(G)+1))}{\delta(G)+1}
$$

Lemma 13 Every graph $G$ has a spanning forest $F$ consisting of trees of order at least $\delta(G)+1$ such that $|R(F)| \leq 2 \gamma(G)-c_{F}$.

Proof. Let $D \subseteq V(G)$ be a dominating set of $G$ of size $\gamma(G)$ and set $N_{v}=\{v\} \cup N_{G}(v)$ for $v \in D$. Define a graph $H$ such that $V(H)=\left\{N_{v}: v \in D\right\}$ and $N_{v} N_{u} \in E(H)$ iff $N_{v} \cap N_{u} \neq \emptyset$ and $v \neq u$ (hence $N_{v} \neq N_{u}$ since $D$ is the smallest dominating set of $G$ ). Let $H_{1}, \ldots, H_{m}$ be the connected components of $H$ and let $T_{1}, \ldots, T_{m}$ be their respective spanning trees. Let $G_{i}=G\left[\bigcup_{N_{v} \in V\left(H_{i}\right)} N_{v}\right]$ and $D_{i}=\left\{v: N_{v} \in V\left(H_{i}\right)\right\} \subseteq D$, $i=1, \ldots, m$. Clearly, each $G_{i}$ is connected, $\left|G_{i}\right| \geq \delta(G)+1, D_{i}$ is a dominating set of $G_{i}$ and $V\left(G_{1}\right) \cup \ldots \cup V\left(G_{m}\right)=V(G), D_{1} \cup \ldots \cup D_{m}=D$. The desired forest will consist of spanning trees of these vertex disjoint subgraphs $G_{i}$ of $G$ which we construct in the following manner. Take e.g. $G_{1}$. Subsequently, for each $u, v \in D_{1}$ such that $N_{u} N_{v} \in E\left(T_{1}\right)$ choose a vertex $w \in N_{u} \cap N_{v}$ and add to the tree the edges $u w$ (if possible, i.e. unless $u=w$ or $u w$ is already in the tree) and $v w$ (if possible). Then we have already constructed a subtree of $G_{1}$ with the vertex set $D_{1}^{\prime}$ such that $D_{1} \subseteq D_{1}^{\prime}$ and $\left|D_{1}^{\prime}\right| \leq 2\left|D_{1}\right|-1$. Since $D_{1}$ is a dominating set of $G_{1}$, we can now join each vertex from $V\left(G_{1}\right) \backslash D_{1}^{\prime}$ with a vertex from $D_{1}$ by an edge and thus construct a spanning tree $F_{1}$ of $G_{1}$ such that $\left|R\left(F_{1}\right)\right| \leq 2\left|D_{1}\right|-1$. After repeating this process for each $G_{i}$ we obtain a spanning forest $F$ (consisting of the trees $\left.F_{1}, \ldots, F_{m}\right)$ of $G$ with $|R(F)| \leq 2 \gamma(G)-c_{F}$.

Lemma 14 Let $G$ be a graph of order $n$ and with $\delta(G) \geq 2$. Then there is a $\left\{P_{2}, P_{3}, P_{3}^{o}\right\}$ factor of $G$ consisting of at most $\frac{n}{\delta(G)+1} P_{3}$ 's and with less than $4 \gamma(G)$ vertices in $P_{2}$ 's and $P_{3}$ 's.

Proof. Let $F$ be a spanning forest of $G$ with components $F_{1}, \ldots, F_{c_{F}}$ such that $|R(F)| \leq$ $2 \gamma(G)-c_{F}$ and $\left|F_{i}\right| \geq \delta(G)+1 \geq 3, i=1, \ldots, c_{F}$. We process the trees $F_{1}, \ldots, F_{c_{F}}$ one after another, so let $T$ be an arbitrary one of them. Let $u$ be a vertex of degree one in this tree, where $N_{T}(u)=\{w\}$, and let us root this tree at $u$. Let $L_{0}, L_{1}, \ldots, L_{k}$ be the sets of vertices on the consecutive levels of this rooted tree, i.e. $L_{i}$ consists of the vertices at distance $i$ from $u$. Then $L_{0}=\{u\}, L_{1}=\{w\}$ and $L_{k} \subseteq L(T)$. We say that a vertex $u_{1} \in V(T)$ is below (above) a vertex $u_{2} \in V(T)$ in $T$ if $u_{1}\left(u_{2}\right)$ lies on the path joining $u_{2}$ $\left(u_{1}\right)$ with $u$ in $T$ and $u_{1} \neq u_{2}$. We will "cut out" the elements of the desired factor from this tree by the following algorithm. Process the levels of the vertices one after another in the reversed order, starting at the level $L_{k-1}$. On a given level, process its vertices one after another in an arbitrary order. Let $T_{0}:=T$ and let $T_{i}$ denote the tree that remains of 
$T_{i-1}$ after processing the consecutive vertex. At the moment we start processing a vertex, the only vertices left above it in the tree are its neighbours. Assume now that we have just created $T_{j}$ and $v \in V(T)$ is the next vertex to be processed. Denote by $X=\left\{x_{1}, \ldots, x_{p}\right\}$ the set of neighbours of $v$ in $T_{j}$ that are above $v$ (hence $X$ consists exclusively of leaves of $T_{j}$ ). Then cut off $\left\lfloor\frac{|X|}{2}\right\rfloor P_{3}^{o}$ 's of the form $x_{l} v^{o} x_{l+1}$ from $T_{j}$ (by removing the vertices $x_{l}, x_{l+1}$ and the edges $x_{l} v, x_{l+1} v$ from $T_{j}$ ) one after another and include them as the components of the factor that we want to create. If there is still a vertex in $X$, say $x_{p}$, cut off $x_{p} v$ (and remove the edge joining $v$ with its neighbour below) as one $P_{2}$ to the factor. The only exception to that last rule occurs if $v=w$ (and $|T|$ is odd), when instead of adding $x_{p} w$, we add $P_{3}=x_{p} w u$ to the factor.

Clearly, each $P_{2}$ and $P_{3}$ of the created $\left\{P_{2}, P_{3}, P_{3}^{o}\right\}$-factor of $T$ must contain at least one vertex from $R(T)$. Since there is at most one $P_{3}$ in this factor, these $P_{2}$ 's and $P_{3}$ may contain at most $2|R(T)|+1$ vertices. By repeating this process for all $F_{i}$ we create a $\left\{P_{2}, P_{3}, P_{3}^{o}\right\}$-factor of $G$ with at most

$$
\sum_{1 \leq i \leq c_{F}}\left(2\left|R\left(F_{i}\right)\right|+1\right)=2|R(F)|+c_{F} \leq 2\left(2 \gamma(G)-c_{F}\right)+c_{F}<4 \gamma(G)
$$

vertices in $P_{2}$ 's and $P_{3}$ 's, and consisting of at most $c_{F} P_{3}$ 's. Since $\left|F_{i}\right| \geq \delta(G)+1$ for $i=1, \ldots, c_{F}$, then $c_{F} \leq \frac{n}{\delta(G)+1}$.

Lemma 15 Let $G$ be a d-regular graph of order $n, d \geq 25$, and let $L=\left\{-\left\lceil\frac{4 n}{d}\right\rceil, \ldots,\left\lceil\frac{4 n}{d}\right\rceil\right\}$. Then there is such an $L$-weighting $g$ of $G$ that the obtained vertex weights are all in $L$ $(g(V) \subseteq L)$ and neither of the vertex weights appears more than $\left\lceil\frac{d}{8}\right\rceil$ times $\left(m_{g} \leq\left\lceil\frac{d}{8}\right\rceil\right)$.

Proof. Let $G$ be a $d$-regular graph of order $n, d \geq 25$, and let $L^{+}=\left\{1, \ldots,\left\lceil\frac{4 n}{d}\right\rceil\right\}$, hence $\left|L^{+}\right|=\left\lceil\frac{4 n}{d}\right\rceil$. Note that $\left\lceil\frac{d}{8}\right\rceil \geq 4$. Let us find a $\left\{P_{2}, P_{3}, P_{3}^{o}\right\}$-factor of $G$ which satisfies the thesis of Lemma 14. Let $A, B, C$ be the sets of $P_{2}$ 's, $P_{3}$ 's, $P_{3}^{o}$ 's, respectively, from this factor. Denote $a:=|A|, b:=|B|$ and $c:=|C|$, hence $2 a+3 b+2 c=n$. By Lemma 14, $b \leq \frac{n}{d+1}$ and $2 a+3 b \leq 4 \gamma(G)$. Therefore, by $(6)$,

$$
c \geq \frac{n}{2}-2 \gamma(G) \geq \frac{n}{2}-2 \frac{n(1+\ln (d+1))}{d+1} .
$$

Note that

$$
f(d):=\frac{1}{2}-2 \frac{1+\ln (d+1)}{d+1}-\frac{4}{d} \geq 0,
$$

since $f$ is an increasing function for $d>0$ and $f(25)>0(f(25) \approx 0,012)$. By $(7),(8)$ and the fact that $c$ is an integer, we have

$$
c \geq\left\lceil\frac{4 n}{d}\right\rceil
$$

Set $g(e)=0$ for each edge $e$ of $G$ outside the factor. Now we will weight the edges of the graphs of the factor one after another. Each time we weight an edge, we establish the 
final weight of at least one (closed) vertex. To ensure that, for each $P_{3}^{o}$ from $C$, its two edges must be weighted by a pair of weights $(j,-j) \in L \times L$, so that the weight of the open vertex remained unchanged.

First we deal with the graphs from $B$. If $b$ is odd (in particular, if $b=1$ ), weight the edges of the first $P_{3}$ by 1 and -1 , hence establish the weights of its three (closed) vertices as 1,0 and -1 . Then, one after another, alternately assign the pair of weights $\left(\left\lceil\frac{n}{2 d}\right\rceil+i, 2\left\lceil\frac{n}{2 d}\right\rceil+i\right)$ and $\left(-\left\lceil\frac{n}{2 d}\right\rceil-i,-2\left\lceil\frac{n}{2 d}\right\rceil-i\right), i=0,1,2, \ldots$, to the pairs of edges of the consecutive $P_{3}$ 's from $B$. This way the vertices of the given $P_{3}$ will obtain weights $\left\lceil\frac{n}{2 d}\right\rceil+i, 2\left\lceil\frac{n}{2 d}\right\rceil+i, 3\left\lceil\frac{n}{2 d}\right\rceil+2 i$ or $-\left\lceil\frac{n}{2 d}\right\rceil-i,-2\left\lceil\frac{n}{d}\right\rceil-i,-3\left\lceil\frac{n}{2 d}\right\rceil-2 i$. Note that for $b \geq 2$ we have

$$
\frac{n}{2 d}>\frac{1}{2} \frac{n}{d+1} \geq \frac{1}{2} b \geq 1
$$

consequently $\left\lceil\frac{n}{2 d}\right\rceil \geq 2$. Moreover, since

$$
2\left\lceil\frac{n}{2 d}\right\rceil \geq \frac{n}{d}>\frac{n}{d+1} \geq b
$$

then $i \leq\left\lceil\frac{n}{2 d}\right\rceil-1$. Therefore, the established so far weights of vertices are all different. Denote the set of these weights by $U$. Note also that if $u \in U$ then $-u \in U$. Finally, by (10), for $b \geq 2$,

$$
3\left\lceil\frac{n}{2 d}\right\rceil+2\left(\left\lceil\frac{n}{2 d}\right\rceil-1\right) \leq 5\left(\frac{n}{2 d}+1\right)-2=\frac{5}{2} \frac{n}{d}+3<4 \frac{n}{d} \leq\left\lceil\frac{4 n}{d}\right\rceil,
$$

hence in all cases we get $U \subseteq L$.

Now we weight the edges of some part of the graphs from $C$. Subsequently, for the elements of $C$, set weights $(i,-i)$ to the pairs of their edges (establishing the weights of their closed vertices as $i$ and $-i$ ) either for all $i \in U \cap L^{+}$if $\left\lceil\frac{d}{8}\right\rceil$ is even or for $i \in L^{+} \backslash U$ if $\left\lceil\frac{d}{8}\right\rceil$ is odd (by (9), there is enough elements in $C$ ). This way, each vertex weight from the set $L \backslash\{0\}$ can still be used an even number of times (up to the total of $\left\lceil\frac{d}{8}\right\rceil$ ).

Now we weight subsequently all $P_{2}$ 's from $A$. First, alternately set 1 and -1 as the weights of the edges from $A$ (each time establishing the weights of two vertices as 1 or -1 ) until there is at most $\left\lceil\frac{d}{8}\right\rceil$ vertices with established weight -1 (and at most $\left\lceil\frac{d}{8}\right\rceil$ vertices with weight 1). Then, alternately set 2 and -2 as the edge weights of the elements from $A$ until there is at most $\left\lceil\frac{d}{8}\right\rceil$ vertices weighted with -2 . Continue so (with $3,4, \ldots$ ) until all the edges in $A$ have been weighted.

At this point a weight $i$ is established for the same number of vertices as $-i$, for $i \in L^{+}$, with one possible exception - for odd $a$, when for one $j \in L^{+}$two more vertices carry the weight $j$ than $-j$. Therefore, we may easily finish the weighting of the elements from $C$. Subsequently, for the remaining graphs in $C$, set weights $(i,-i), i \in L^{+}$, to the pairs of their edges as long as possible, i.e. as long as the number of vertices with the established weight $i$ is less than $\left\lceil\frac{d}{8}\right\rceil$ for some $i \in L^{+}$and as long as there are still some elements of $C$ left unweighted. At the end either all the edges are already weighted and the weighting obtained complies with our requirements or there are still some elements of $C$ left unweighted. In the second case however, by our construction, we must have already 
weighted at least $2\left\lceil\frac{4 n}{d}\right\rceil\left\lceil\frac{d}{8}\right\rceil-2 \geq n-2$ vertices (this " -2 " may occur only if $a$ is odd). Therefore, at most one $P_{3}^{o}$ from $C$ remained unweighted. Then we may weight its edges with 0 , establishing the weight of the two remaining vertices as 0 . This way, at most 3 vertices (together with at most one from the first part of the proof concerning $B$ ) have weight 0 . Since $\left\lceil\frac{d}{8}\right\rceil \geq 3$, the construction is complete.

Proof of Theorem 5. Let $G$ be a $d$-regular graph, $d \geq 2$, of order $n$ (hence $d<n$ ).

Assume first that $d \leq 25$. Then by Theorem 2 ,

$$
\begin{aligned}
s(G) & \leq\left\lceil\frac{n}{2}\right\rceil+9 \leq \frac{n}{2}+\frac{1}{2}+9=\frac{n d+19 d}{2 d}= \\
& =\frac{32 n+12 d}{2 d}+\frac{n(d-25)+7(d-n)}{2 d}<16 \frac{n}{d}+6 .
\end{aligned}
$$

Let now $d>25$. Then, by Lemma 15, there is such a weighting $g$ of $G$ with numbers from the set $L=\left\{-\left\lceil\frac{4 n}{d}\right\rceil, \ldots,\left\lceil\frac{4 n}{d}\right\rceil\right\}$ that the obtained vertex weights are all in $L$ and neither of the vertex weights appears more than $\left\lceil\frac{d}{8}\right\rceil$ times. Let $A_{1}, \ldots, A_{\left\lceil\frac{d}{8}\right\rceil}$ be a partition of $V(G)$ such that $g(u) \neq g(v)$ if $u, v \in A_{i}$ and $u \neq v, i=1, \ldots,\left\lceil\frac{d}{8}\right\rceil$. Now, by Corollary 11, there is a weighting $f: E(G) \rightarrow\left\{\left\lceil\frac{4 n}{d}\right\rceil+1,3\left\lceil\frac{4 n}{d}\right\rceil+2\right\}$ such that $d_{f}\left(A_{i}, A_{j}\right) \geq 2\left\lceil\frac{4 n}{d}\right\rceil+1$ for $i \neq j$ and $d_{f}\left(A_{i}\right)=0$ or $d_{f}\left(A_{i}\right) \geq 2\left\lceil\frac{4 n}{d}\right\rceil+1$ for all $i$. It is easy to see that a weighting $f+g$ is irregular for $G$. Therefore, since $(f+g): E(G) \rightarrow\left\{1, \ldots, 4\left\lceil\frac{4 n}{d}\right\rceil+2\right\}$, we have

$$
s(G) \leq 4\left\lceil\frac{4 n}{d}\right\rceil+2<16 \frac{n}{d}+6 .
$$

Acknowledgements. I wish to express my thanks to Felix Lazebnik for bringing the main problem of this paper to my attention during an interesting discussion in Budapest, and to Ingo Schiermeyer for his help and remarks in Freiberg. I also enclose greetings for the anonymous referee for their valuable comments and suggestions. The author informs that his research were supported by MNiSzW grant no. N N201 389134.

\section{References}

[1] L. Addario-Berry, K. Dalal, B.A. Reed, Degree constrained subgraphs, Proceedings of GRACO2005, volume 19 of Electron. Notes Discrete Math., Amsterdam (2005) 257-263 (electronic), Elsevier.

[2] N. Alon, J.H. Spencer, The Probabilistic Method, John Wiley and Sons, Inc., 1992.

[3] G. Chartrand, M.S. Jacobson, J. Lehel, O.R. Oellermann, S. Ruiz, F. Saba, Irregular networks. Proc. of the 250th Anniversary Conf. on Graph Theory, Fort Wayne, Indiana (1986).

[4] B. Cuckler, F. Lazebnik, Irregularity Strength of Dense Graphs, to appear in J. Graph Theory. 
[5] R.J. Faudree, J. Lehel, Bound on the irregularity strength of regular graphs, Colloq Math Soc Jańos Bolyai, 52, Combinatorics, Eger North Holland, Amsterdam (1987) 247-256.

[6] A. Frieze, R.J. Gould, M. Karoński, F. Pfender, On Graph Irregularity Strength, J. Graph Theory 41 (2002) no. 2 120-137.

[7] J. Lehel, Facts and quests on degree irregular assignments, Graph Theory, Combinatorics and Applications, Willey, New York (1991) 765-782. 